(1)

CrossMark

\title{
A long way to go after the initial experience with balloon pulmonary angioplasty
}

\author{
Hiromi Matsubara ${ }^{1,2}$ and Aiko Ogawa ${ }^{1}$
}

Affiliations: ${ }^{1}$ Dept of Clinical Science, National Hospital Organization Okayama Medical Center, Okayama, Japan. ${ }^{2}$ Dept of Cardiology, National Hospital Organization Okayama Medical Center, Okayama, Japan.

Correspondence: Hiromi Matsubara, Depts of Clinical Science and Cardiology, National Hospital Organization Okayama Medical Center, 1711-1 Tamasu, Kita-ku, Okayama 701-1192, Japan.

E-mail: matsubara.hiromidgmail.com

@ERSpublications

To improve efficacy and safety of balloon pulmonary angioplasty, accumulation of experience and knowledge is needed http://ow.ly/lVSj30bFFNh

Cite this article as: Matsubara H, Ogawa A. A long way to go after the initial experience with balloon pulmonary angioplasty. Eur Respir J 2017; 49: 1700718 [https://doi.org/10.1183/13993003.00718-2017].

To date, the standard treatment for chronic thromboembolic pulmonary hypertension (CTEPH) has been surgical pulmonary endarterectomy (PEA) followed by life-time anticoagulation therapy [1]. However, excellent results with PEA have been limited to select centres with experience and expertise; many patients who are possible candidates for PEA at expert centres would be considered ineligible in many countries [2]. Medical treatment has shown little or no improvement in haemodynamic parameters in patients with CTEPH who are ineligible for PEA $[3,4]$. Thus, despite partial success of balloon pulmonary angioplasty (BPA) in the USA [5], attempts to treat patients with CTEPH by BPA have increased in several countries where PEA is not widely performed [6-8]. Before starting a BPA programme, two main problems in the initial report [5] need to be resolved: its low efficacy and high incidence of complication.

First, to improve the efficacy of BPA, it is necessary for it to have the same treatment goal as PEA: complete removal of obstructive intraluminal debris from the entire pulmonary arterial circulation [9]. Thus, during our initial BPA experience, we tried to completely dilate as many stenotic or obstructive lesions as possible. As expected, the decrease in mean pulmonary arterial pressure correlated with the number of treated segments [6]. An average of 12 segments was treated in each patient [6], which was double the number in the initial report from the USA [5]. Accordingly, the reduction in mean pulmonary arterial pressure was also double [6] that in the initial report [5]. Meanwhile, recent reports have failed to decrease the occurrence of complication, i.e. pulmonary injury, after BPA [6-8]. The main cause of pulmonary injury was initially believed to be reperfusion oedema, but attempts to prevent the occurrence of pulmonary oedema by using steroid treatment or positive airway pressure were ineffective in preventing pulmonary injury after BPA [6]. However, there was a learning curve in preventing pulmonary injury after BPA [6], indicating that the main cause of pulmonary injury after BPA is not the same as that of reperfusion oedema after PEA. We evaluated the initial 500 procedures and found that angiographically apparent vascular injury was observed in 107 out of 130 procedures leading to pulmonary injury after BPA [10]. The leading cause of complication was distal vascular injury to the lesion caused by the tip of the guidewire, and most of the other causes were due to overdilation of the lesion. It seems clear that the main cause of pulmonary injury after BPA is not reperfusion oedema but haemorrhage caused by iatrogenic vascular injury.

Received: April 052017 | Accepted: April 062017

Conflict of interest: Disclosures can be found alongside this article at erj.ersjournals.com

Copyright @ERS 2017 
In this issue of the European Respiratory Journal, OLsson et al. [11] report the first German experience with BPA, and confirm that the most common complications after BPA were pulmonary vascular injury and consecutive pulmonary bleeding. Compared with initial reports $[6,7]$, they succeeded in reducing the occurrence of complication, which was probably the result of careful manipulation of the guidewire and the selection of undersized balloons [11]. The complication rate in Bad Nauheim, Germany, where the intervention was guided only with conventional fluoroscopy, was similar to that in Hanover, Germany, where selective C-arm computed tomography (CT) was used to select a target lesion. Special diagnostic tools, such as selective C-arm CT are ineffective in reducing complications, thus simple pulmonary angiography may be sufficient for starting a BPA programme.

Unfortunately, haemodynamic improvement was not pronounced in the reported study. The authors discuss reasons for the low efficacy of their BPA procedures compared with that of initial reports, and suggest that differences in patient population and disease duration caused their low efficacy [11]. Their cohort of inoperable patients had a different background from the previous cohort treated with PEA at the same centre [12], e.g. patients were more than 10 years older and predominantly women. However, the patients' demographic characteristics were very similar to that of the Japanese cohort of inoperable patients $[6,7]$, even though this cohorts might have included some patients who would be deemed operable in Germany. Although OLsSON et al. [11] stated that their patients had a relatively long disease duration, the average interval between the CTEPH diagnosis and first BPA was 3.2 years in the initial report from Japan [6], which is not shorter than that in the current report. The reason for the small haemodynamic improvement may be that OLsson et al. [11] tried to reduce the complication rate only by using undersized balloons, which may diminish the effect of BPA. Therefore, the use of undersized balloons with the other strategies being unchanged would diminish the efficacy of the entire procedure.

Compared with medical treatment, BPA requires expensive devices and repeated admission and it is also an invasive procedure with radiation. Therefore, normalisation of haemodynamic parameters should be the minimum aim when performing BPA, otherwise, there is no advantage of BPA over medical treatment [13]. Then, how can BPA operators improve the efficacy of BPA while maintaining safety? One solution for this conflicting issue may be dilating the same lesions that have already been treated with undersized balloons a second time, and increasing repetition of the procedures. This would normalise blood flow in the treated segments by dilating the lesion to the optimal diameter. However, it would not increase the number of treated lesions. Another solution would be to increase the number of treated segments by increasing the repetition of procedures with undersized balloons. However, undersized dilatation would not normalise blood flow in treated segments. Moreover, both possible solutions require a higher cost and increase the risk of radiation. Increasing the number of lesions treated in a single procedure would be the only feasible solution. To improve the efficacy of BPA, increasing the number of treated segments and treating difficult lesions are important. Although obstructive lesions are difficult to treat and it is easy to cause vascular injury [10], the haemodynamic improvement achieved by treating obstructive lesions, if possible, is expected to be larger than that achieved by targeting stenotic lesions.

Treating many lesions, including difficult lesions, within a limited time without causing complications will not be particularly easy, but it is possible. In the initial 255 procedures performed at our institution, a median of three vessels per procedure were treated [6], and this increased to four vessels per procedure after experience with 500 procedures [10]. After experience with more than 1000 procedures, this number further increased to achieve our current treatment goal of a mean pulmonary artery pressure of $<25 \mathrm{mmHg}$ and oxygen saturation $>95 \%$ without using any vasodilators or oxygen [13]. Of note, no serious complications after BPA were seen during the most recent 700 procedures. As is the case with PEA, an excellent result with catheter-based BPA seems limited to select centres with experience and expertise. Although OLsson et al. [11] state that their experience is sufficient, the number of procedures at each centre was only about half of the number of experienced procedures in the initial report [6]. MADANI et al. [14] demonstrated that haemodynamic improvements in the initial cases with PEA were less than those of the recent cases. The accumulation of experience and knowledge regarding BPA for CTEPH is needed after the initial experience. The accumulation of experience of BPA can be expected at European pulmonary hypertension centres, unlike in Japan, where regulation does not exist and more than 70 hospitals are performing BPA. In the near future, the experience and technique of European BPA operators will catch up to those of Japanese operators. This will be the time to start a prospective, international, multicentre study of BPA to elucidate its safety and efficacy and the long-term survival of patients.

\section{References}

1 Galiè N, Humbert M, Vachiery JL, et al. 2015 ESC/ERS Guidelines for the diagnosis and treatment of pulmonary hypertension: The Joint Task Force for the Diagnosis and Treatment of Pulmonary Hypertension of the European Society of Cardiology (ESC) and the European Respiratory Society (ERS): Endorsed by: Association for European 
Paediatric and Congenital Cardiology (AEPC), International Society for Heart and Lung Transplantation (ISHLT). Eur Heart J 2016; 37: 67-119.

2 Pepke-Zaba J, Delcroix M, Lang I, et al. Chronic thromboembolic pulmonary hypertension (CTEPH): results from an international prospective registry. Circulation 2011; 124: 1973-1981.

3 Jensen KW, Kerr KM, Fedullo PF, et al. Pulmonary hypertensive medical therapy in chronic thromboembolic pulmonary hypertension before pulmonary thromboendarterectomy. Circulation 2009; 120: 1248-1254.

4 Ghofrani HA, Hoeper MM, Halank M, et al. Riociguat for chronic thromboembolic pulmonary hypertension and pulmonary arterial hypertension: a phase II study. Eur Respir J 2010; 36: 792-799.

5 Feinstein JA, Goldhaber SZ, Lock JE, et al. Balloon pulmonary angioplasty for treatment of chronic thromboembolic pulmonary hypertension. Circulation 2001; 103: 10-13.

6 Mizoguchi H, Ogawa A, Munemasa M, et al. Refined balloon pulmonary angioplasty for inoperable patients with chronic thromboembolic pulmonary hypertension. Circ Cardiovasc Interv 2012; 5: 748-755.

7 Kataoka M, Inami T, Hayashida K, et al. Percutaneous transluminal pulmonary angioplasty for the treatment of chronic thromboembolic pulmonary hypertension. Circ Cardiovasc Interv 2012; 5: 756-762.

8 Andreassen AK, Ragnarsson A, Gude E, et al. Balloon pulmonary angioplasty in patients with inoperable chronic thromboembolic pulmonary hypertension. Heart 2013; 99: 1415-1420.

9 Madani M, Mayer E, Fadel E, et al. Pulmonary endarterectomy. Patient selection, technical challenges, and outcomes. Ann Am Thorac Soc 2016; 13 Suppl 3: S240-S247.

10 Kawakami T, Ogawa A, Miyaji K, et al. Novel angiographic classification of each vascular lesion in chronic thromboembolic pulmonary hypertension based on selective angiogram and results of balloon pulmonary angioplasty. Circ Cardiovasc Interv 2016; 9: e003318.

11 Olsson K, Wiedenroth CB, Kamp J-C, et al. Balloon pulmonary angioplasty for inoperable patients with chronic thromboembolic pulmonary hypertension: the initial German experience. Eur Respir J 2017; 49: 1602409.

12 Rolf A, Rixe J, Kim WK, et al. Right ventricular adaptation to pulmonary pressure load in patients with chronic thromboembolic pulmonary hypertension before and after successful pulmonary endarterectomy--a cardiovascular magnetic resonance study. J Cardiovasc Magn Reson 2014; 16: 96.

13 Kawakami T, Ogawa A, Miyaji K, et al. Response by Kawakami et al to letter regarding article, "novel angiographic classification of each vascular lesion in chronic thromboembolic pulmonary hypertension based on selective angiogram and results of balloon pulmonary angioplasty". Circ Cardiovasc Interv 2017; 10: e004962.

14 Madani MM, Auger WR, Pretorius V, et al. Pulmonary endarterectomy: recent changes in a single institution's experience of more than 2,700 patients. Ann Thorac Surg 2012; 94: 97-103. 